\title{
Future proton-oxygen beam collisions at the LHC for air shower physics
}

\author{
Hans P. Dembinski* \\ Max Planck Institute for Nuclear Physics, Heidelberg, Germany \\ E-mail: hdembins@mpi-hd.mpg.de
}

Ralf Ulrich

Karlsruhe Institute of Technology, Karlsruhe, Germany

E-mail: ralf.ulrichekit.edu

\section{Tanguy Pierog}

Karlsruhe Institute of Technology, Karlsruhe, Germany

E-mail: tanguy.pierog@kit.edu

\begin{abstract}
We present a study of the impact of future measurements of proton-oxygen collisions at the LHC on air shower observables. The LHC has successfully demonstrated its capability to deliver ion beams in combination with protons in its first two runs, successfully injecting lead and xenon. In 2018, the second run ended and the science case for the coming runs with a high-luminosity LHC was developed by a large collaboration of CERN physicists and published as a Yellow Report in December 2018. Ion beams remain an important topic, but the scientific focus is on heavy ions and symmetric configurations like lead-lead, which are not ideal to address current challenges in cosmic ray research. However, this report also contains the motivation for measuring proton-oxygen collisions for air shower physics and a recommendation to have one week of beam time allocated in 2023 to this topic during Run 3. Here, we report on these development, which are important for the future of cosmic ray research and the scientific arguments from the study. We show that nuclear effects are important and that measuring proton-proton and proton-lead collisions is not sufficient to understand air showers. In particular, the energy fraction which goes into neutral pions could play an important role in solving the Muon Puzzle in ultra-high energy air showers.
\end{abstract}

36th International Cosmic Ray Conference -ICRC2019-

July 24th - August 1st, 2019

Madison, WI, U.S.A.

${ }^{*}$ Speaker. 


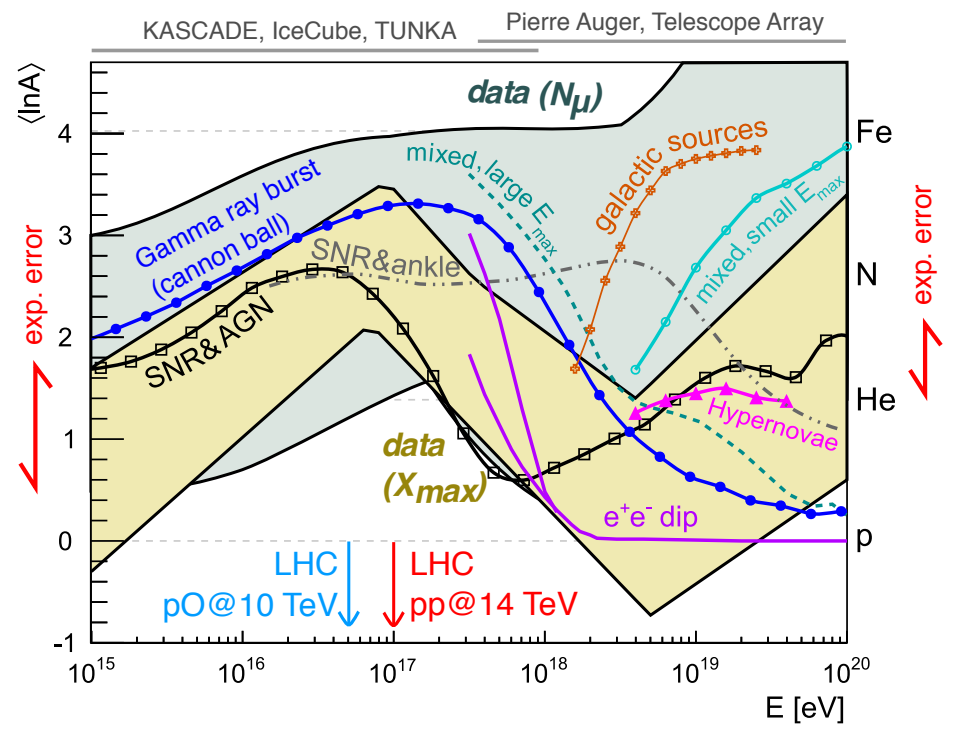

Figure 1: Mass composition of cosmic rays quantified by $\langle\ln A\rangle$ as a function of cosmic ray energy $E[3$, section 11.3]. See Ref. [4] for references to data (bands) and model predictions (markers and lines), and the text for a discussion.

\section{Introduction}

High-energy cosmic rays are messengers of extreme astrophysical phenomena in the universe. The recent coincident observation of gamma rays and neutrinos from the flaring blazar TXS 0506+056 [1] confirmed that active galactic nuclei produce high-energy cosmic rays. Cosmic rays as messenger particles reach higher energies than gamma rays and have a detection efficiency of nearly $100 \%$, much higher than neutrinos. However, cosmic rays are charged and bent onto unpredictable paths by galactic and intergalactic magnetic fields. Large-scale anisotropies are observed [2], but attempts to directly observe sources have been unsuccessful.

Nevertheless, the diffusive flux of cosmic rays carries an important imprint of the sources in the mass composition of cosmic rays as a function of their energy. This is illustrated in Fig. 1, which displays predictions (lines and markers) of the mean $\operatorname{logarithmic}$ mass $\langle\ln A\rangle$ for several source scenarios. Measurement of $\langle\ln A\rangle$ can severely constrain these scenarios and exclude theories.

Above particle energies of about $10^{15} \mathrm{eV},\langle\ln A\rangle$ can only be indirectly inferred from extensive air showers initiated by cosmic rays in Earth's atmosphere. One has to simulate air showers to connect observable features of the shower with the mass of the cosmic ray. The two main observables are the depth $X_{\max }$ of the shower maximum in the atmosphere represented by the yellow band in Fig. 1, and the number $N_{\mu}$ of muons produced in the shower represented by the green band. The width of those bands has two main contributions: the experimental uncertainties and the hadronic model uncertainties inherent in converting the air shower observables into $\langle\ln A\rangle$.

Leading experiments achieve instrumental accuracies of $10 \%$ of the proton-iron difference for measuring the observables. This would strongly discriminate between source scenarios, but the air shower simulations that are required to convert the observables to $\langle\ln A\rangle$ add a large model uncertainty. The simulations use the multi-purpose heavy-ion event generator EPOS-LHC [5], or specialized generators for air showers, like QGSJet-II.04 [6] and SIBYLL-2.3c [7]. The generators are designed to describe hadron-nucleus and nucleus-nucleus interactions and soft-QCD phenomena like diffraction and forward production, which are very important for the air shower development. The generators combine Regge field theory tuned to available data and perturbative 
QCD. Uncertainties in these models arise from a lack of data on the production of light hadrons in the very forward direction in hadron-nucleus interactions at the $\mathrm{TeV}$ scale.

On the one hand, LHC measurements have already significantly improved the model predictions for $X_{\max }$ in the latest generation of models. This improvement stems mainly from highprecision measurements of the inelastic cross-section (see e.g. [8] and references therein). Further measurements of hadron rapidity spectra now have the potential to reduce the remaining spread below $10 \%$ which is the experimental accuracy. On the other hand, the model spread for $N_{\mu}$ is still large and predictions are not consistent with $X_{\max }$ for cosmic rays with the same mass.

Recently, eight air shower experiments combined their data on muon measurements and found $8 \sigma$ evidence for a discrepancy between the data and state-of-the-art air shower simulations [ 9 , and references therein]. The muon abundance rises not fast enough with the shower energy in simulations, leading to an underestimation of the muon abundance starting at about $10^{16} \mathrm{eV}$. This corresponds to a center-of-mass energy of $4.3 \mathrm{TeV}$, which is well accessible by the LHC. The origin of this discrepancy is theoretically not well understood and attempts to resolve it by a simple retuning of the hadronic generators have been unsuccessful. This is called the Muon Puzzle.

Solving the Muon Puzzle and resolving the ambiguity in the cosmic ray mass composition is a strategic goal in the cosmic ray field for the coming decade [10]. In the following, we report on a proposal to accelerate an oxygen beam at the LHC during Run 3 [3, section 12] and to measure proton-oxygen collisions, supported by leading members of the LHCb and CMS collaborations. These measurements have the potential to resolve the Muon Puzzle and to reduce the uncertainty in $X_{\max }$ predictions to a negligible amount. Quantitative predictions of the potential impact of these measurements are presented. We identified rapidity spectra of light hadrons as well as the ratio of electromagnetic and hadronic energy flow extending well into the forward direction as the most important measurements at the LHC to improve air shower simulations.

\section{Impact of hadronic interactions features at the LHC on air shower observables}

Evaluating the impact of changes in hadronic interaction features that can be measured in colliders on air shower observables is technically challenging. Air showers are formed by a cascade of hadronic interactions at exponentially decreasing center-of-mass energies. The first hadronic interaction plays an important role for $X_{\max }$ and the fluctuations of the muon number [12], but not for the average number of muons produced. Muons originate mainly from decays of charged pions after several (5 to 10) hadronic interactions. Their number and energy distribution is shaped by the convolved effect of these hadronic interactions. This makes it difficult to pinpoint the microscopic cause of deviations between simulation and experiment.

Nevertheless, progress in this direction has been made. The Heitler-Matthews model [13] provides a simple picture of an air shower cascade, which allows one to make rough predictions for the impact of hadronic interaction features on air shower observables. More reliable predictions have been obtained with special Monte Carlo simulations [14], in which the standard predictions of a hadronic generator were artificially modified in an energy-dependent way. Two aspects of multi-particle production with a strong effect on $N_{\mu}$ have been identified: the hadron multiplicity $N_{\text {mult }}$, and the energy fraction $\alpha$ that goes into neutral pions. If the average number of hadrons per interaction increases, then the muon number increases. Some energy in each interaction is carried 

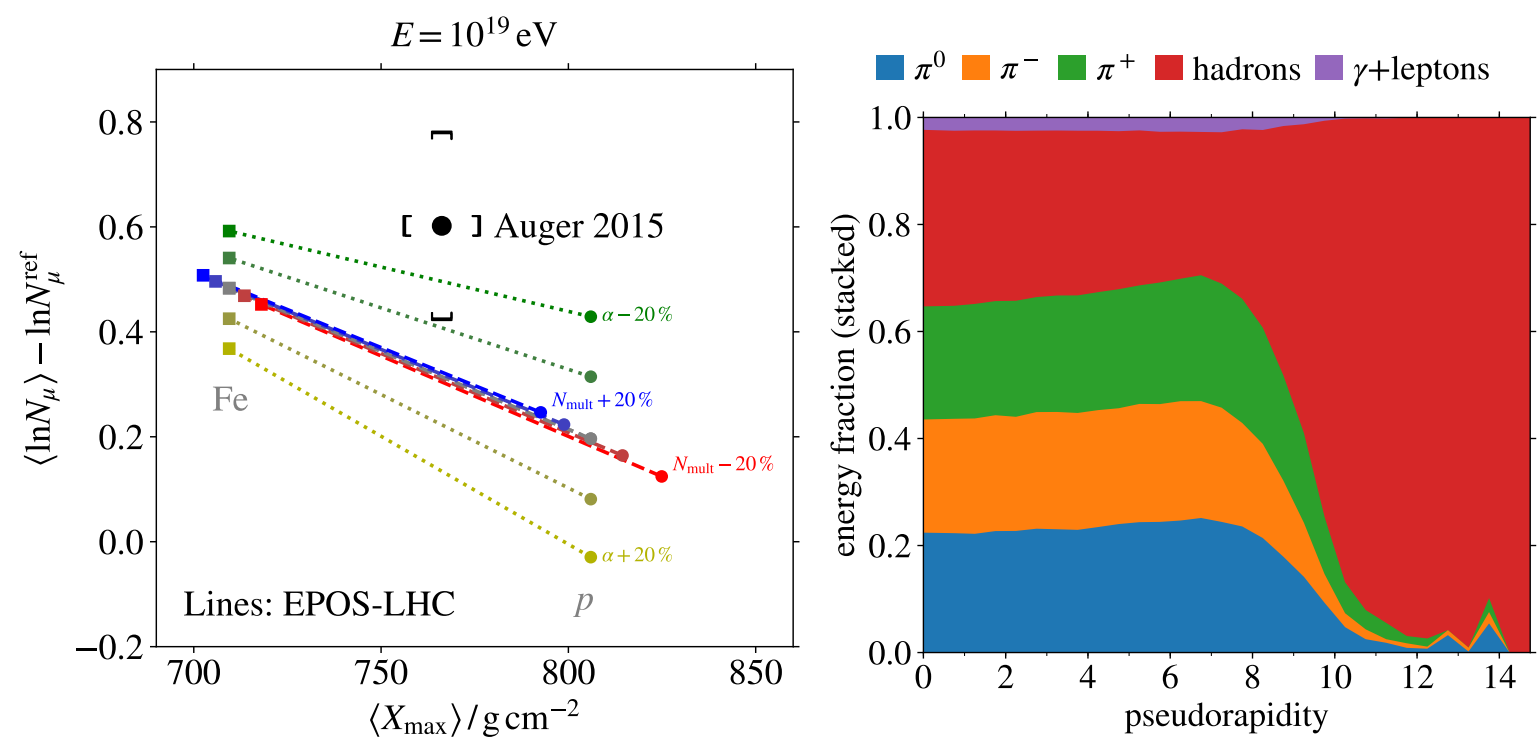

Figure 2: Left: Impact of changes of the hadron multiplicity $N_{\text {mult }}$ (dashed lines) and the energy fraction $\alpha$ (dotted lines) which goes into neutral pions in collisions at the LHC energy scale on EPOS-LHC predictions for $X_{\max }$ and $\ln N_{\mu}$ in $10^{19} \mathrm{eV}$ air showers, the (representative) data point is from the Pierre Auger Observatory [11]. The model lines represent all values that can be obtained with any mixture of cosmic nuclei from proton (bottom right) to iron (top left). The dashed and dotted lines represent modifications of $N_{\text {mult }}$ and $\alpha$ in steps of $\pm 10 \%$ from their nominal values. Plot taken from Ref. [3, section 11.3]. Right: Fraction of energy carried by different particle species as a function of pseudorapidity in pp collisions at $\sqrt{s}=13 \mathrm{TeV}$ simulated with EPOS-LHC. With the exception of neutral pions, hadrons with a life-time smaller than $3 \mathrm{~ns}$ are set to decay in the simulation. Most of the energy in the very forward region is carried by hadrons which are not pions.

by neutral pions which immediately decay into gamma rays, their energy is lost for the hadronic cascade. If less energy is lost in this way, more muons are produced.

The impact of changing these variables in EPOS-LHC at $\sqrt{s}=13 \mathrm{TeV}$ and extrapolating the change upward in energy is illustrated in Fig.2, left-hand-side, for showers of $10^{19} \mathrm{eV}$. Changing the hadron multiplicity $N_{\text {mult }}$ changes both $X_{\max }$ and the number of muons in such a way that the gap to the data cannot be closed, since the model line only shifts parallel to itself. Measuring $N_{\text {mult }}$ is important to make the uncertainty of $X_{\max }$ predictions negligible, this alone is not sufficient to resolve the Muon Puzzle. The most promising solution for the Muon Puzzle is a reduction of $\alpha$, the fraction of energy that goes into neutral pions in each interaction. A reduction of $15 \%$ at the LHC energy scale (and correspondingly more at higher energies) would resolve the Muon Puzzle.

While the number ratios of charged to neutral pions are fixed to about 2:1 by isospin symmetry, there are also other hadrons produced in a hadronic interaction, mostly kaons, protons and neutrons, which carry a significant fraction of the energy, as illustrated by Fig.2, right-hand-side. Especially in the very forward region at large rapidity, which is most important for air shower development, a lot of energy is not carried by pions.

Several mechanisms which effectively reduce $\alpha$ have been proposed. Strangeness production in hadron-nucleus collisions may be larger than anticipated [15], this would effectively reduce $\alpha$, as a higher fraction of energy is carried by kaons. Evidence for an enhancement of multi-strange 

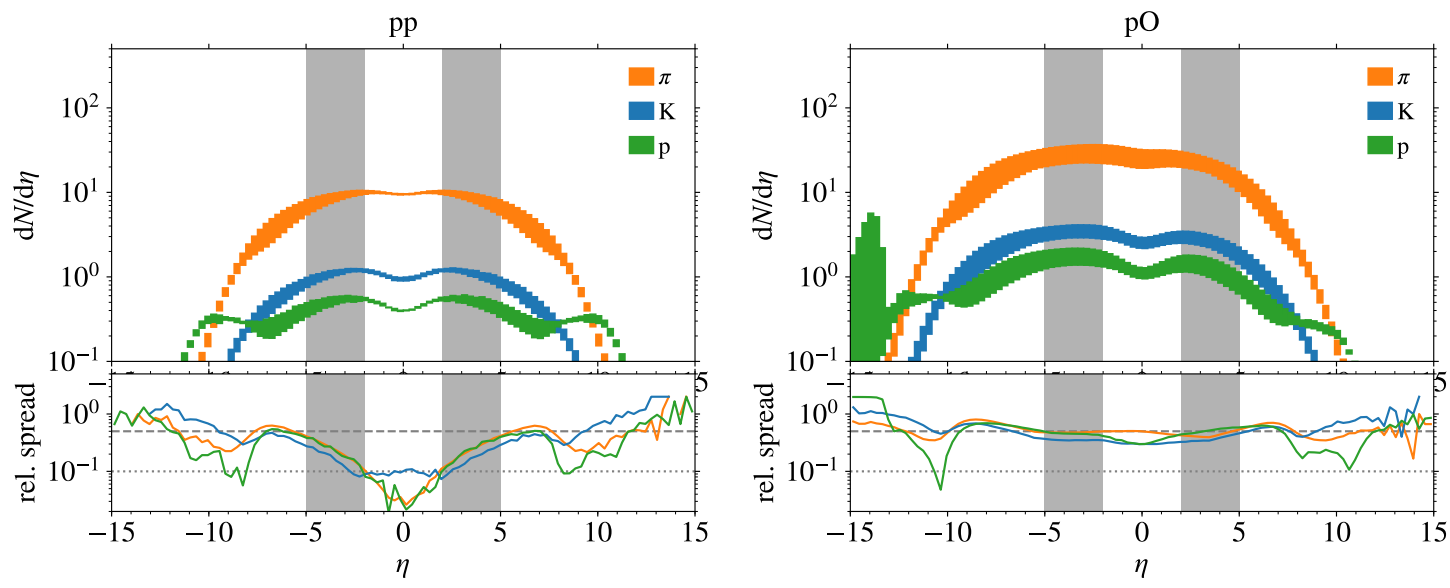

Figure 3: Simulation of pseudorapidity spectra of charged pions, charged kaons, and protons in pp at $13 \mathrm{TeV}$ and p-O collisions at $10 \mathrm{TeV}$ with CRMC [23]. The width of the bands indicates the spread of the generators EPOS-LHC [24], QGSJet-II.04 [6], and SIBYLL-2.3 [7]. The lower plots show the relative spread of the bands. The dotted (dashed) line indicates $10 \%(50 \%)$ spread. Gray regions indicate the pseudorapidity range covered by the LHCb experiment. Plot taken from Ref. [3, section 11.3].

baryons in high-multiplicity events was recently reported by the ALICE collaboration [16]. The EPOS-LHC model predicts collective effects in hadron-nucleus collision that reduce pion production at mid-rapidity $[17,18]$. It is also possible that the rapidity spectrum of pions is narrower than anticipated, so that the relative pion yield at forward rapidity is lower compared to kaons, protons, and neutrons. These theoretical possibilities need to be constrained with data.

\section{Nuclear modification effects and impact of proton-oxygen measurements}

The most common hadronic interaction in an air shower is between a hadron and a nitrogen or oxygen nucleus. Accelerating oxygen ions is feasible in the LHC and a proposal has been accepted to measure $\mathrm{p}-\mathrm{O}$ collisions during Run 3 in 2023 [3, section 12]. The p-O system is very attractive for reference measurements, since it is close to interaction systems which actually occur in air showers. The pp system is already used to tune the hadronic generators, but it is not sufficient.

For particles produced into the direction of the proton, a p-O collision cannot be approximated as a superposition of independent pp collisions. For a particle to be produced in the direction of the proton, the momentum contributed by the parton from the nucleus is low, which means it has a large de Broglie wavelength. This leads to interference which modifies the interaction outcome compared to a system where the nucleus is treated as a superposition of free nucleons. This effect is well known. It has been observed at the LHC in several measurements $[19,20,21]$ and explains the differences observed in air showers simulated in a hydrogen atmosphere [22].

The generators implement this effect differently and extrapolate differently to $\mathrm{p}-\mathrm{O}$ from the pp reference system that is mainly used for tuning. This is illustrated by Fig. 3, in which the spread of the predicted pseudorapidity spectra of light hadrons is shown. The agreement is good for pp collisions at mid-rapidity, where the models are tuned to LHC data, but the spread reaches $50 \%$ in the forward region. In p-O collisions the spread is about $50 \%$ everywhere, even at midrapidity. The difficulty of predicting light ion collisions is further illustrated in Fig. 4. EPOSLHC predictions for Xe-Xe collisions significantly underestimate the observed yields in the central 

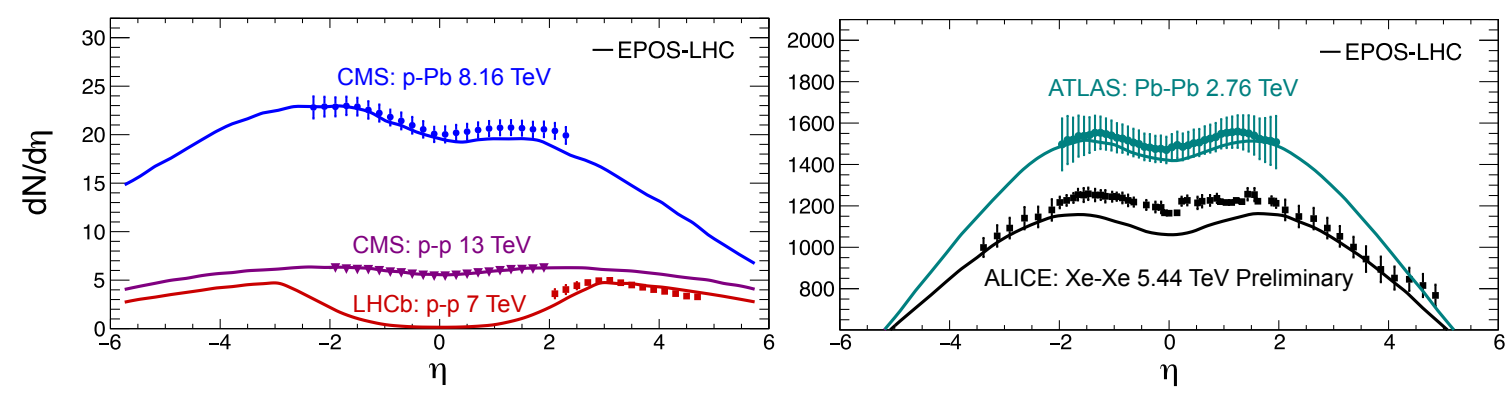

Figure 4: Comparison of charged particle multiplicity measurements at different center-of-mass energies and in different colliding systems with the EPOS-LHC model [25]. Shown in both plots is $\mathrm{d} N / \mathrm{d} \eta$. Plot taken from Ref. [3, section 11.3].

region, despite a satisfactory description of $\mathrm{pp}, \mathrm{p}-\mathrm{Pb}$, and $\mathrm{Pb}-\mathrm{Pb}$ collisions. The deviations in $\mathrm{Xe}-\mathrm{Xe}$ are much larger than what is expected from a simple interpolation [25].

The possibility to constraining $\alpha$ and $N_{\text {mult }}$ to $5 \%$ without p-O collisions was investigated, but is very challenging. Selecting peripheral $\mathrm{p}-\mathrm{Pb}$ collisions to mimic $\mathrm{p}$-air collisions with the same number of binary collisions was considered as an alternative, but such a measurement cannot reach the required accuracy. Centrality in $\mathrm{p}-\mathrm{Pb}$ collisions is extracted from the data using various centrality estimators with different selection biases. These biases would increase the uncertainty of the proposed measurements well beyond the target of $5 \%$ [26]. On the contrary, p-O measurements could provide a sensitive test of centrality estimators since the thickness of the oxygen nucleus and hence the average number of wounded nucleons is about a factor of two smaller.

Finally, the results in $\mathrm{pp}, \mathrm{p}-\mathrm{O}$, and $\mathrm{p}-\mathrm{Pb}$ collisions should be systematically compared. If the results can be shown to follow a simple scaling law, then the accuracy of the p-O predictions could be further improved with existing and future data from $\mathrm{p}-\mathrm{Pb}$ collisions. Currently, it is not clear whether a simple scaling law exists. The dominant nuclear effects are expected to be different for collisions of protons with light and heavy nuclei. A lead-nucleus has a much smaller surface-tovolume-ratio than an oxygen-nucleus and much larger electric fields, which cause non-negligible photo-hadron interactions in peripheral collisions.

\section{Proposed measurements at the LHC}

Accelerating protons and oxygen beams at the LHC with a rigidity of 13 TV yields a centerof-mass energy of about $10 \mathrm{TeV}$ per nucleon. For air showers, measuring light hadrons produced in minimum-bias events in the direction of the proton is crucial. Planned is one week of data taking [3, section 12], in which $2 \mathrm{nb}^{-1}$ of luminosity for proton-oxygen can be achieved, which is 10 times more than the minimum necessary to achieve a statistical accuracy of $5 \%$ for pseudorapidity spectra of charged pions, charged kaons, and protons.

The ALICE and LHCb experiments can measure these spectra to a total accuracy of $5 \%$ in a pseudorapidity range $-0.9<\eta<5$. Both ALICE and LHCb have detectors for particle identification. Our projections show that a measurement of this accuracy could reduce the uncertainty of $X_{\max }$ predictions below $10 \%$, making it negligible compared to the experimental uncertainty. The measurements also have the clear potential to resolve the muon puzzle by strongly constraining the 
fraction $\alpha$ of energy carried by neutral pions. The neutral pion spectrum is constrained by measurements of the charged pion spectra. Similarly, the spectra of charged kaons and protons constrain neutral kaons and neutrons. Altogether, these particles carry most of the energy of the interaction. In particular, the baryon spectrum was found to be important for the muon abundance, despite the overall comparably small number of baryons compared to pions [27].

The LHCf experiment is able to detect neutral pions and neutrons at $\eta>8.4$ and can constrain $\alpha$ in the very forward phase space. The CMS and ATLAS detectors have no particle identification capabilities, but can constrain $\alpha$ and $N_{\text {mult }}$ by seperately measuring the flows of electromagnetic and hadronic energy in the respective calorimeter systems. The energy deposit in the electromagnetic calorimeter comes almost entirely from neutral pion decays, while the hadronic calorimeter measures the energy carried by other hadrons. The CMS experiment with CASTOR can cover $-6.6<\eta<5.2$ and ATLAS $-4.5<\eta<4.5$. The sum of energy flows constrains $N_{\text {mult }}$ and their ratio constrains $\alpha$ very efficiently.

\section{Conclusions and outlook}

Measurements of $\mathrm{p}-\mathrm{O}$ collisions at the LHC are crucial to resolve the ambiguity in the cosmicray mass composition. Measurements of identified hadron spectra and energy flows have the potential to reduce the uncertainty of $X_{\max }$ predictions below $10 \%$ and to resolve the Muon Puzzle. This in turn will resolve the ambiguity in the mass-composition of high-energy cosmic rays and severely constrain theories on cosmic-ray origins. Data taking is planned for one week in 2023, in which $2 \mathrm{nb}^{-1}$ of integrated luminosity are to be accumulated. Comparing the same measurements in $\mathrm{pp}, \mathrm{p}-\mathrm{O}$, and $\mathrm{p}-\mathrm{Pb}$ will determine how hadron spectra and energy flows change with the number of nucleons in the nucleus.

A period of oxygen acceleration in the SPS would also provide the opportunity to complement cosmic-ray related measurements of nuclear fragmentation at NA61/SHINE[28, 29] at beam momenta of $150 \mathrm{AGeV} / \mathrm{c}$. These measurements aim at improving our understanding of the cosmic-ray propagation in the Galaxy and to evaluate the cosmic-ray background for signatures of astrophysical dark matter [30]. Another opportunity is the study of very forward production of hadrons in the p-O system at $\sqrt{s_{N N}} \sim 100 \mathrm{GeV}$ at the LHCb experiment, by colliding the oxygen beam with proton gas provided by an upgraded SMOG system [3, section 11.4].

\section{References}

[1] Liverpool Telescope, MAGIC, H.E.S.S., AGILE, Kiso, VLA/17B-403, INTEGRAL, Kapteyn, Subaru, HaWC, Fermi-LAT, ASAS-SN, VERITAS, Kanata, ICECube, SWift NUSTAR collaboration, Science 361 (2018) eaat1378 [1807. 08816].

[2] Pierre Auger collaboration, Astrophys. J. 804 (2015) 15 [1411. 6111].

[3] Z. Citron et al., Future physics opportunities for high-density QCD at the LHC with heavy-ion and proton beams, Tech. Rep. CERN-LPCC-2018-07, CERN, Geneva, 2018 [1812. 06772].

[4] K.-H. Kampert and M. Unger, Astropart. Phys. 35 (2012) 660 [1201. 0018].

[5] K. Werner, F.-M. Liu and T. Pierog, Phys. Rev. C74 (2006) 044902 [hep-ph / 0506232 ].

[6] S. Ostapchenko, Phys. Rev. D83 (2011) 014018 [1010 . 1869]. 
[7] F. Riehn, H. P. Dembinski, R. Engel, A. Fedynitch, T. K. Gaisser and T. Stanev, The hadronic interaction model SIBYLL 2.3c and Feynman scaling, 35th International Cosmic Ray Conference (ICRC 2017): Bexco, Busan, Korea, July 12-20, 2017, POS ( ICRC2017) 301 (2017).

[8] LHCB collaboration, JHEP 06 (2018) 100 [1803. 10974 ].

[9] EAS-MSU, ICECUbe, KASCADE-Grande, NEVOD-DECOR, PIERRE Auger, SUGAR, TElESCOPE ArRAY, YAKUTSK EAS ARRAY collaboration, EPJ Web Conf. 210 (2019) 02004 [1902.08124].

[10] F. G. Schröder et al., Bull. Am. Astron. Soc. 51 (2019) 131 [1903.07713].

[11] Pierre Auger collaboration, Phys. Rev. D91 (2015) 032003 [1408. 1421].

[12] F. Riehn for the PIERRE AUGER collaboration, Measurement of the fluctuations in the number of muons in inclined air showers with the Pierre Auger Observatory, 36th International Cosmic Ray Conference (ICRC 2019): Madison, Wisconsin, USA, July 25 - August 1, 2019, POS (ICRC2019) 404 (2019).

[13] J. Matthews, Astropart. Phys. 22 (2005) 387.

[14] R. Ulrich, R. Engel and M. Unger, Phys. Rev. D83 (2011) 054026 [1 010 . 4310].

[15] L. A. Anchordoqui, H. Goldberg and T. J. Weiler, Phys. Rev. D95 (2017) 063005 [1612.07328].

[16] ALICE collaboration, Nature Phys. 13 (2017) 535 [1606.07424].

[17] S. Baur, H. Dembinski, T. Pierog, R. Ulrich and K. Werner, The ratio of electromagnetic to hadronic energy in high energy hadron collisions as a probe for collective effects, and implications for the muon production in cosmic ray air showers, arXiv:1902.09265 (2019) [1902 . 09265].

[18] T. Pierog, S. Baur, H. Dembinski, R. Ulrich and K. Werner, Collective hadronization and air showers: can LHCdata solve the muon puzzle?, 36th International Cosmic Ray Conference (ICRC 2019): Madison, Wisconsin, USA, July 25 - August 1, 2019, POS ( ICRC2019) 387 (2019).

[19] LHCB collaboration, Phys. Lett. B774 (2017) 159 [1706. 07122].

[20] ALICE collaboration, JHEP 11 (2018) 013 [1802.09145].

[21] LHCF collaboration, Phys. Rev. D94 (2016) 032007 [1507. 08764 ].

[22] D. d'Enterria, T. Pierog and G. Sun, Impact of QCD jets and heavy-quark production in cosmic-ray proton atmospheric showers up to $10^{20} \mathrm{eV}$, arXiv:1809.06406 (2018) [1809.06406].

[23] T. Pierog, C. Baus and R. Ulrich, $C R M C$, web.ikp.kit.edu/rulrich/crmc.html.

[24] T. Pierog et al., Phys. Rev. C92 (2015) 034906 [1306.0121].

[25] ALICE collaboration, ALICE results on system-size dependence of charged-particle multiplicity density in $\mathrm{p}-\mathrm{Pb}, \mathrm{Pb}-\mathrm{Pb}$ and $\mathrm{Xe}$-Xe collisions, in 27th International Conference on Ultrarelativistic Nucleus-Nucleus Collisions (Quark Matter 2018) Venice, Italy, May 14-19, 2018, 2018, 1807.09061.

[26] ALICE collaboration, Phys. Rev. C91 (2015) 064905 [1412.6828].

[27] T. Pierog and K. Werner, Phys. Rev. Lett. 101 (2008) 171101 [astro-ph/0611311].

[28] NA61/SHINE collaboration, Feasibility Study for the Measurement of Nuclear Fragmentation Cross Sections with NA61/SHINE at the CERN SPS, Tech. Rep. CERN-SPSC-2017-035, SPSC-P-330-ADD-9, CERN, Geneva, Oct, 2017.

[29] NA61/SHINE collaboration, Study of Hadron-Nucleus and Nucleus-Nucleus Collisions at the CERN SPS: Early Post-LS2 Measurements and Future Plans, Tech. Rep. CERN-SPSC-2018-008, SPSC-P-330-ADD-10, CERN, Geneva, Mar, 2018.

[30] Y. Genolini, D. Maurin, I. V. Moskalenko and M. Unger, Phys. Rev. C98 (2018) 034611 [1803.04686]. 